\title{
PREVALÊNCIA, ÍNDICES DE INFECÇÃO E HÁBITOS ALIMENTARES DE TRIATOMÍNEOS CAPTURADOS EM UMA ÁREA DE VIGILÂNCIA EPIDEMIOLÓGICA
}

\author{
Vera Lúcia C. Corrêa Rodrigues, Antenor do Nascimento Ferraz Filho, \\ Eduardo Olavo da Rocha e Silva e Virgilia Luna Castor de Lima
}

\begin{abstract}
Em continuação a estudo anterior, relativo à prevalência e infecção por Trypanosoma cruzi dos triatomineos capturados na região administrativa de Campinas, SP, os autores apresentam dados do periodo de 1982-1986, acrescentando informações sobre respastos sangüineos realizados por 7.785 exemplares. Para tanto, foram utilizados os anti-soros: ave, marsupial, roedor e humano, através dos quais constataram o ecletismo alimentar de Panstrongylus megistus, espécie predominante na região, cujas formas aladas são encontradas com freqüencia, infectadas por $\mathrm{T}$. cruzi, nas casas habitadas. Desses, $14,78 \%$ reagiramfrente ao anti-soro humano. Com Rhodnius neglectus foi observada situação assemelhada, mas com nümeros menos expressivos. Em relação ao Triatoma sordida, não foi constatada infecção natural e tampouco sinais de ingestão de sangue humano. Foi constatado acentuado aumento de $\mathrm{T}$. arthurneivai nas casas, fruto da provável modificação ocorrida no ambiente natural. No período, foi encontrado Microtiatoma borbai, detectado pela primeira vez no Estado de São Paulo. Ressaltam também a importância da "investigação de foco" nas áreas em fase de vigilância.
\end{abstract}

Palavras-chaves: Triatomíneos. Trypanosoma cruzi. Hábito alimentar. Panstrongylus megistus. Microtriatoma borbai.

Trabalhos relacionados com triatomíneos coletados em municípios pertencentes à Região Administrativa de Campinas-SP, não são raros na literatura especializada, em parte devido ao laboratório de Chagas em Mogi Guaçu, um dos setores de pesquisa da Superintendência de Controle de Endemias (SUCEN), da Secretaria de Estado de Saúde do Governo do Estado de São Paulo, mas sobretudo pela presença do $P$ anstrongylus megistus em processo de domiciliação muito bem estudado por Forattini e cols ${ }^{9} 1011$. Vale lembrar que o fenômeno da domiciliação do $P$. megistus, segundo Forattini $^{5}$, é conseqüência das modificações antrópicas acontecidas na paisagem local que, de coberta (florestas do domínio atlântico), vem sendo transformada em paisagem aberta.

Recentemente, Ferraz Filho e Rodrigues ${ }^{3}$ publicaram um estudo sobre a distribuição e

Superintendência de Controle de Endemias (SUCEN) da Secretaria da Saúde, Mogi Guaçu, SP, Brasil.

Endereço para correspondência: Dra. Vera Lúcia C. Corrêa Rodrigues. Laboratório de Chagas/SUCEN. Rua Afonso Pessini 86, Centro. CP: 192, 13840-970 Mogi Guaçu, SP, Brasil.

Recebido para publicação em 21/07/92. prevalência dos triatomíneos naquela região, com informações adicionais sobre a infecção por Trypanosoma cruzi, abrangendo o período 19741981. Retornam agora, trazendo dados referentes ao período 1982-1986, procurando enfocar também o tipo de repasto realizado pelos 7.785 triatomíneos examinados.

O trabalho procura mostrar que as atividades de controle nesta área devem obrigatoriamente incluir medidas apropriadas ao estudo dos focos ${ }^{13}$, particularmentenas localidades onde ocorrem espécies em domiciliação, com exemplares infectados pelo $T$. cruzi, em contato com o homem -áreas em vigilância.

\section{MATERIAL E MÉTODOS}

Os triatomíneos estudados resultaram em boa parte das atividades de captura de "barbeiros", levadas a efeito pelas equipes de campo do Serviço Regional 5 - Campinas (SUCEN - SR-5), no trabalho rotineiro de cobertura anual das localidades programadas entre 1982 e 1986 e, de outra parte, das "denúncias de focos", realizadas pelos moradores com subsequente atendimento da denúncia pela 
Rodrigues VLCC, Ferraz Fịlho AN, Silva EOR, Lima VLC. Prevalência, índices de infecção e hábitos alimentares de triatomíneos capturados em uma área de vigilância epidemiológica. Revista da Sociedade Brasileira de Medicina Tropical 25:183-190, jul-set, 1992.

SUCEN. Foram vasculhadas à procura de triatomíneos, casas habitadas, casas desabitadas e/ ou abandonadas; peridomicílios com os respectivos anexos e em situações julgadas de interesse, $o$ ambiente silvestre próximo às casas.

Os exemplares capturados, devidamente registrados, eram encaminhados ao Laboratório de Chagas em Mogi Guaçu, para identificação e realização dos exames programados. Oexame inicial, efetuado a partir da compressão abdominal, visava a retirada do material fecal necessário à pesquisa de tripanosomatídeos. Constatada sua presença a fresco, eram então preparadas lâminas finas que, depois do material fixado e seco, era corado pelo método May-Grunwald e Giemsa, para correta identificação. Em alguns casos, escolhidos aleatoriamente, foram efetuadas inoculações em camundongos e ratos albinos, para posterior elaboração de cortes histológicos dos órgãos mais freqüentemente sujeitos à infecção chagásica.

$\mathrm{Na}$ realização dos testes de precipitina empregou-se o método estabelecido por Siqueira ${ }^{16}$, para identificação do sangue ingerido pelos triatomíneos. Para tanto, o material abdominal era recolhido em papel de filtro, devidamente identificado. Os anti-soros utilizados foram elaborados a partir de coelhos, submetidos a uma série de inoculações de antígenos (antiave, antimarsupial, anti-roedor e anti-humano) até ser alcançado o título adequado.

Cada uma das 7.785 amostras, foi colocada frente a uma bateria formada pelos quatro tipos de anti-soros acima considerados, exceção feita às do T. arthurneivai que, em função do freqüente achado junto a lacertídeos (Tropidurus), teve incluído na bateria $o$ anti-soro de lagarto.

\section{RESULTADOS E DISCUSSÃO}

A Tabela 1 apresenta os totais de triatomíneos, por espécie, examinados pelo Laboratório de Mogi Guaçu, no período 1982-1986. De acordo com esta tabela, o total geral de triatomíneos examinados, procedentes de diversas localidades situadas na Região Administrativa de Campinas, foi de 7.785 exemplares. A Tabela 2 mostra os infectados por $T$. cruzi, observados entre os examinados. Exemplares de cinco espécies se apresentaram com a infecção natural, das quais, $P$. megistus e $R$. neglectus ${ }^{15} \mathrm{com}$
Tabela 1 - Totalde triatomíneos examinados por espécie da Região Administrativa de Campinas. Laboratório de Chagas - Mogi Guaçul SUCEN; São Paulo. 1982 a 1986.

\begin{tabular}{lrr}
\hline Espécie & $\begin{array}{c}\mathrm{n}^{0} \text { de } \\
\text { exemplares }\end{array}$ & $\%$ \\
\hline Panstrongylus megistus & 6.951 & 89,28 \\
Triatoma sordia & 357 & 4,58 \\
Rhodnius neglectus & 276 & 3,54 \\
Triatoma arthurneivai & 134 & 1,72 \\
Psammolestes tertius & 33 & 0,42 \\
Triatoma infestans & 14 & 0,17 \\
Panstrongylus diasi & 9 & 0,11 \\
Panstrongylus geniculatus & 8 & 0,10 \\
Microtriatoma borbai & 3 & 0,03 \\
\hline Total & 7.785 & 100,00
\end{tabular}

índices expressivos e presença, como veremos à frente, no interior das moradias de molde a merecer atenção. Vale recordar que Ferraz Filho e Rodrigues $^{3}$, trabalhando com material procedente da mesma região, referente ao período 1974-1981, identificaram as mesmas espécies de triatomíneos, com exceção de Microtriatoma borbai, cujo encontro ocorreu pela primeira vez no Estado de São Paulo, por dois dos autores deste trabalho ${ }^{4}$, no período agora estudado. Como ocorreu no periodo anterior, $P$. megistus foi também aqui a espécie predominante, respectivamente $91,3 \%$ e $89,28 \%$. Em relação a $T$. sordida, ocorreu um aumento relativo no número de exemplares coletados que, de $2,49 \%$ no período $74-81$, passou agora para $4,58 \%$ do total geral. Em compensação, o índice de infecção pelo $T$. cruzi caiu para zero. Embora em números absolutos a presença do $R$. neglectus seja menor neste trabalho, em números relativos aumentou, pois passou de $1,65 \%$ para $3,54 \%$ do total dos examinados. Aconteceu também uma pequena queda no índice de infecção da espécie que, de $6,65 \%$ em 618 examinados, caiu para $5,43 \%$ em 276 exemplares. T. arthurneivai, nos dois períodos estudados se manteve no patamar de 1,72\% dos examinados. Um exemplar da espécie foi encontrado com infecção por $T$. cruzi. Ressaltamos ser o segundo achado em T. arthurneivai, uma vez que o primeiro registro ocorreu no período 1974-19813. O número de exemplares coletados das demais espécies foi 
Rodrigues VLCC, Ferraz Filho AN, Silva EOR, Lima VLC. Prevalência, índices de infecção e hábitos alimentares de triatomíneos capturados em uma área de vigilância epidemiológica. Revista da Sociedade Brasileira de Medicina Tropical 25:183-190, jul-set, 1992.

Tabela 2 - Infecção por T. cruzi, segundo a espécie do triatomíneo e local da captura. Laboratório de Chagas - Mogi Guaçu/SUCEN; São Paulo. 1982 a 1986.

\begin{tabular}{lcrcccc}
\hline Espécie & $\begin{array}{c}\text { Total de } \\
\text { exemplares } \\
\text { capturados }\end{array}$ & $\begin{array}{c}\text { Lasa } \\
\text { habitada }\end{array}$ & $\begin{array}{c}\text { Casa } \\
\text { desabitada }\end{array}$ & $\begin{array}{c}\text { Peridomi- } \\
\text { cílio }\end{array}$ & silvestre & $\begin{array}{c}\text { Total } \\
\text { de } \\
\text { infectados }\end{array}$ \\
\hline Panstrongylus megistus & 6.951 & 157 & 180 & 454 & 49 & $840(12,08 \%)$ \\
Rhodnius neglectus & 276 & 6 & - & 4 & 5 & $15(5,43 \%)$ \\
Triatoma arthurneivai & 134 & - & - & 1 & - & 1 \\
Panstrongylus geniculatus & 8 & 2 & - & - & - & 2 \\
Microtriatoma borbai & 3 & - & - & - & 1 & 1 \\
\hline Total & 7.372 & 165 & 180 & 459 & 55 & $859(11,65 \%)$
\end{tabular}

pequeno, não permitindo maiores comentários, à exceção de $T$. infestans, pela sua importância no passado, reduzida ao encontro de 14 exemplares, nenhum infectado por T. cruzi.

A Tabela 3 expõe os resultados das reações de precipitina frente a cada um dos $6.951 P$. megistus. Notamos inicialmente a presença de maior número de exemplares na fase alada, nas casas habitadas, do que ninfas. Tal fato decorre, possivelmente, do fenômeno da dispersão após a eclosão, quando abandonam os criadouros naturais, localizados, no caso, nas proximidades das casas invadidas. Segundo Forattini e cols ${ }^{11}$, a dispersão da espécie se processa com freqüência maior na região, no último trimestre do ano. Em relação ao tipo de repasto, o que esta tabela apresenta é o ecletismo alimentar do $P$. megistus, onde está inserido o repasto em sangue humano, conseqüência da apreciável valência ecológica da espécie ${ }^{9}$. A invasão dos ecótopos artificiais e em determinadas circunstâncias sua colonização nos mesmos, os índices de infecção natural elevados e ocasionais repastos em sangue humano, são fatores que tornam possível a ocorrência da transmissão. Foram examinados, procedentes de casas habitadas, 157 exemplares infectados pelo $T$. cruzi (Tabela 2), dos quais 115 alados, representando $12,51 \%$ dos $P$. megistus capturados nas moradias. Desses, $17(14,74 \%)$ se mostraram reagentes para sangue humano. Vale também recordar que os indices de infecção por $T$. cruzi se situaram nesses níveis, devido à presença nos criadouros naturais de $P$. megistus, dos didelfídeos e roedores, principais reservatórios de $T$. cruzi em nosso meio ${ }^{112}$.

Embora T. sordida tenha se constituído na segunda espécie em quantidade de exemplares coletados, com 357 examinados, seu significado epidemiológico foi menor, uma vez que a grande maioria foi encontrada em anexos situados no peridomicílio. Quase todos, inclusive aqueles capturados nas casas, se mostraram reagentes apenas para $o$ anti-soro ave (Tabela 4). No período, não foi detectada infecção por T. cruzi em nenhum dos exemplares examinados da espécie.

A Tabela 5 apresenta os dados referentes a $R$. neglectus que, sem o mesmo peso dos números apresentados para $P$. megistus, mesmo assimpermite supor que apresenta ecletismo alimentar. Aqui também o número de alados nas casas habitadas foi bem mais elevado do que das ninfas, ou seja, 120 formas adultas contra 3 ninfas. Uma vez mais, devemos nos reportar a Forattini ${ }^{6}$ para relatar que a dispersão desta espécie acontece de preferência ao longo do primeiro semestre do ano. Vale salientar ainda que os moradores das áreas com espécies em domiciliação estão atentos ao problema, pois na maioria das vezes não permitiram, através das denúncias à SUCEN e/ou ações próprias, a formação de colônias de "barbeiros" em suas residências. Forattini e cols ${ }^{7}$ já relataram que o $R$. neglectus pode colonizar nos ecótopos artificiais, devido ao ecletismo alimentar e destruição do seu habitat natural (cume de palmeiras). Chama à atenção o fato deque entre os exemplaresque, na região, invadiram casas habitadas, 6 (50\% do total) se apresentavam infectados pelo T. cruzi (Tabela 2), sendo 2 deles reagentes para soro anti-humano.

$\mathrm{Na}$ Tabela 6 são apresentadas as informações disponíveis a respeito do $T$. arthurneivai, onde se 
Rodrigues VLCC, Ferraz Filho AN, Silva EOR, Lima VLC. Prevalência, indices de infecção e hábitos alimentares de triatomíneos capturados em uma área de vigilância epidemiológica. Revista da Sociedade Brasileira de Medicina Tropical 25:183-190, jul-set, 1992.

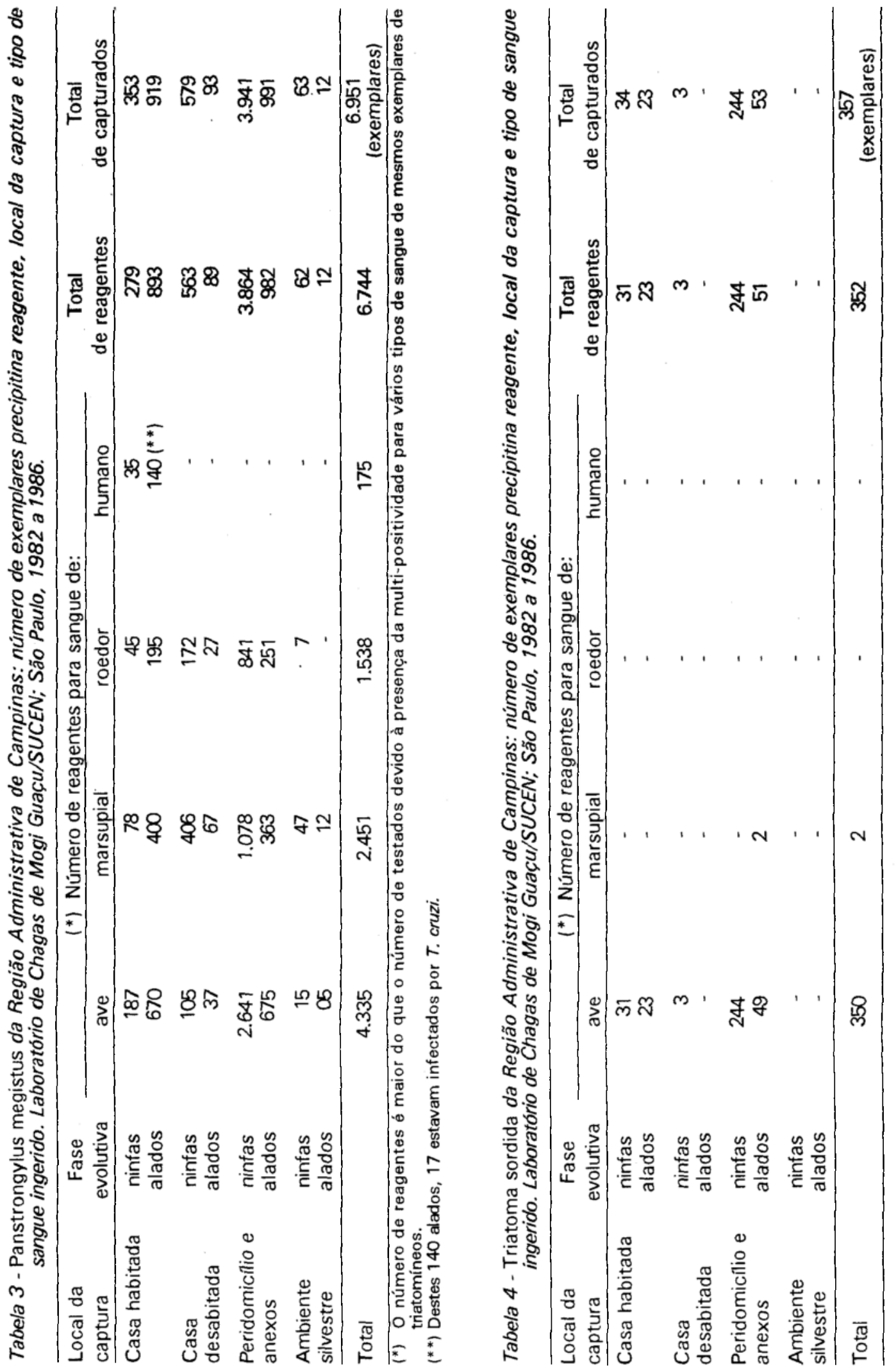


Rodrigues VLCC, Ferraz Filho AN, Silva EOR, Lima VLC. Prevalência, índices de infeç̧ão e hábitos alimentares de triatomineos capturados em uma área de vigilância epidemiológica. Revista da Sociedade Brasileira de Medicina Tropical 25:183-190, jul-set, 1992.

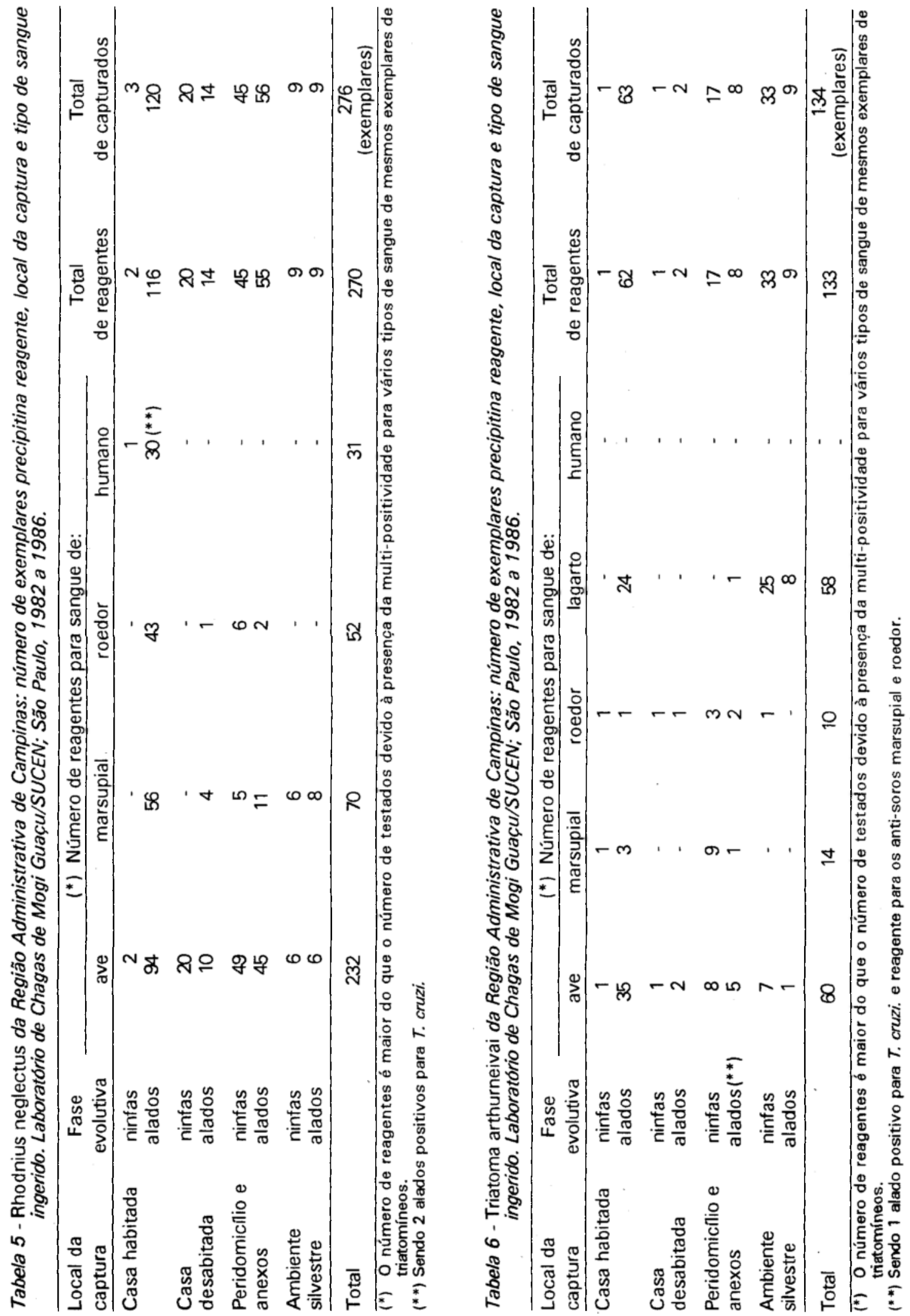


Rodrigues VLCC, Ferraz Filho AN, Silva EOR, Lima VLC. Prevalência, indices de infecção e hábitos a ămueniares de triatomíneos capturados em uma área de vigilância epidemiológica. Revista da Sociedade Brasileira de Medicina Tropical 25:183-190, jul-set, 1992.

Tabela 6.1 - Triatoma arthurneivai, número de exemplares coletados e local da captura. Laboratório de Chagas. Mogi Guaçu/SUCEN; São Paulo.

\begin{tabular}{lcccc}
\hline Período & $\begin{array}{c}\text { Casas } \\
\text { habitadas }\end{array}$ & $\begin{array}{c}\text { Casas } \\
\text { desabitadas }\end{array}$ & $\begin{array}{c}\text { Peridomicílio } \\
\text { e anexos }\end{array}$ & $\begin{array}{c}\text { Total de } \\
\text { exemplares }\end{array}$ \\
\hline $1974-1981$ & $16(25,40 \%)$ & 8 & 39 & 63 \\
$1982-1986$ & $64(69,56 \%)$ & 3 & 25 & 92 \\
\hline
\end{tabular}

pode constatar a importância dos lacertídeos e aves na dieta alimentar da espécie. Segundo Barreto ${ }^{2}$ vale lembrar ainda que ela ocupa criadouros localizados em buracos e fendas existentes em locais pedregosos. Ressalta-se que 63 alados e 1 ninfa $(47,76 \%)$ foram encontrados em casas habitadas, mostrando a espécie, poder invasivo na região maior que $T$. sordida $(15,96 \%) \mathrm{e} R$. neglectus $(44,56 \%)$. Destes 64, nenhum continha sangue humano no tubo intestinal. Independentemente desta última constatação e da grande mobilidade das formas aladas ${ }^{8}$, uma aproximação domiciliar deste porte (Tabela 6.1), contrastando com a observada no período anterior (1974-1981) por Ferraz $\mathrm{F}^{\mathrm{O}}$. e Rodrigues $^{3}$, faz supor significativa alteração no habitat natural.

Em relação às demais espécies, devido ao pequeno número de exemplares coletados, as informaçôes disponíveis seguem abaixo:

\section{Psammolestes tertius}

No período, foram capturados 33 exemplares (24 alados e 9 ninfas). Todos encontrados em ninhos de João-graveto (Furnariidae) e todos sororeagentes para sangue de ave.

\section{Triatoma infestans}

Foram coletados 14 exemplares ( 9 alados e 5 ninfas). Das formas aladas encontradas, 6 exemplares foram capturados em casas habitadas, sendo que 3 deles se mostraram reagentes para sangue humano. Os outros três, reagiram para ave. O restante, 8 exemplares, foi capturado no peridomicnlio (anexos) e se mostraram reagentes para anti-soro ave.

\section{Panstrongylus diasi}

Foram encontrados 9 exemplares, todos na forma alada. Apenas um era procedente do peridomicílio; os 8 restantes foram capturados em casas habitadas. Alguns deles (6) se mostraram soro reagentes para ave e/ou marsupial.

\section{Panstrongylus geniculatus}

Foram coletados 8 exemplares ( 7 alados e 1 ninfa), todos encontrados em casas habitadas. Barreto $^{2}$ e Sherlock ${ }^{14}$ relatam que se trata de espécie de ampla distribuição geográfica, com criadouros situados em locas de animais, especialmente buracos de tatu. Infelizmente, na ocasião não se dispunha de anti-soro tatu. Dos exemplares examinados, apenas um foi soro reagente para sangue humano. Os demais reagiram frente à ave, roedor e/ou marsupial.

\section{Microtriatoma borbai}

Pela primeira vez 3 exemplares foram capturados no Estado de São Paulo, encontrados em ambiente silvestre, em um ninho de didelphídeo (gambá). Dos três, apenas um foi examinado, sendo positivo para $T$. cruzi e reagente para marsupial ${ }^{4}$.

Concluindo, em que pese o desconhecimento de casos autóctones da tripanossomíase americana, na Região Administrativa de Campinas, as informações contidas neste trabalho não são de molde a que se possa descartar totalmente tal possibilidade. No período 1982-1986, ficou clara a existência do contato triatomíneo infectado-homem, pelo encontro de sangue humano no tubo intestinal de alguns desses exemplares. A possibilidade da ocorrência aumenta onde são encontradas espécies em domiciliação, com infecão natural, representada na região por $P$. megistus e de uma maneira bem mais discreta pelo $R$. neglectus.

Os fatos aqui apresentados justificam a manutenção das atividades de controle (vigilância) em algumas localidades da região. 
Rodrigues VLCC, Ferraz Filho AN, Silva EOR, Lima VLC. Prevalência, indices de infecção e hábitos alimentares de triatomineos capturados em uma área de vigilancia epidemiológica. Revista da Sociedade Brasileira de Medicina Tropical 25:183-190, jul-set, 1992.

Devem ter prosseguimento os estudos de acompanhamento dos processos de domiciliação dos triatomíneos, em andamento na região. Outros, como o aumento da presença dos exemplares de $T$. arthurneivai nas moradias, devem ser considerados.

\section{SUMMARY}

Prevalence of triatomines and their infection by Trypanosoma cruzi, were studied during 1974-1981, in the Administrative Region of Campinas, São Paulo, Brazil. This investigation was continued (1982-1986), blood meal tests with bird, marsupial, rodent and human antisera having been added. Panstrongylus megistus, predominant in the region, whose winged instars are often found infected by T. cruzi in inhabited houses, displayed and alimentary eclecticism. From a sample of $7,785,14.78$ percent reacted positively with human antisera. A similar, but less marked behaviour was observed in Rhodnius neglectus. Triatoma sordida, the secondmost important species in the Region, was neither demonstrated to be feeding on humans nor to be infected by $\mathrm{T}$. cruzi. Changes in their natural habitat probably caused sharp rises in $\mathrm{T}$. arthurneivai populations in the human dwellings. The capture of three specimes of Microtriatoma borbai marks the first occurrence of this species in the State São Paulo. "Focus investigation"in areas in the surveillance phase can detect triatomines in the process of domiciliation.

Key-words: Triatominae. Trypanosoma cruzi. Blood meals. Panstrongylus megistus. Microtriatoma borbai.

\section{REFERÊNCIAS BIBLIOGRÁFICAS}

1. Barreto MP. Estudos sobre reservatórios e vetores silvestres do Trypanosoma cruzi. XXI-Observações sobre as associações entre reservatórios e vetores em especial referência à região nordeste do Estado de São Paulo. Revista Brasileira de Biologia 28:481494, 1968.

2. Barreto MP. Epidemiologia. In: Brener Z, Andrade ZA (ed) Trypanosoma cruzi e doença de Chagas. Editora Guanabara Koogan, Rio de Janeiro p.89$151,1979$.

3. Ferraz Filho AN, Rodrigues VLCC. Distribuição e infeç̧ão natural de triatomíneos capturados na região de Campinas, São Paulo, Brasil. Revista da Sociedade Brasileira de Medicina Tropical 20:25-30, 1987.

4. Ferraz Filho AN, Rodrigues VLCC. The first encounter of Microtriatoma borbai (Hemiptera: Reduviidae), in the State of São Paulo, Brazil. In: Abstracts ofXIII Annual Meeting on Basic Research in Chagas' Disease, Caxambu p.VE-166, 1986.

5. Forattini OP. Biogeografia, origem e distribuição da domiciliação de triatomíneos no Brasil. Revista de Saúde Pública, São Paulo 14:265-299, 1980.

6. Forattini OP, Ferreira OA, Rabello EX, Barata JMS, Santos JLF. Aspectos ecológicos da trypanossomíase americana. XVIII Desenvolvimento e ciclos anuais de colônias de Triatoma infestans, Triatoma sordida e Rhodnius neglectus em ecótopos artificiais, no ambiente peri e extradomiciliar. Revista de Saúde Pública, São Paulo 17:243-261, 1983.
7. Forattini OP, Rabello EX, Ferreira OA, Rocha e Silva EO, Santos JFL. Aspectos ecológicos da trypanossomíase americana. XXI - Comportamento de espécies de triatomíneos silvestres na reinfestação do intra e peridomicílio. Revista de Saúde Pública, São Paulo 18:185-208, 1984.

8. Forattini OP, Rabello EX, Pattoli DBG. Aspectos ecológicos da trypanossomíase americana. IV Motilidade do Triatoma arthurneivai em ecótopos artificiais. Revista de Saúde Pública, São Paulo 6:183-187, 1972.

9. Forattini OP, Rocha e Silva EO, Ferreira OA, Rabello EX, Santos JLF, Lima AR. Aspectos ecológicos da trypanossomíase americana. XI Domiciliação do Panstrongylus megistus e potencial enzoótico. Revista de Saúde Pública, São Paulo 11:527-550, 1977.

10. Forattini OP, Rocha e Silva EO, Rabello EX, Andrade JCR, Rodrigues VLCC. Aspectos ecológicos da trypanossomíase americana. XIII Potencial de Panstrongylus megistus sob vigilância epidemiológica. Revista de Saúde Pública, São Paulo 12:417-424, 1978.

11. Forattini OP, Santos JLF, Ferreira OA, Rocha e Silva EO, Rabello EX. Aspectos ecológicos da trypanossomíase americana. XVI - Dispersão e ciclos anuais de colônias de Triatoma sordida e de Panstrongylus megistus espontaneamente desenvolvidas em ecótopos artificiais. Revista de Saúde Pública, São Paulo 13:299-313, 1979. 
Rodrigues VLCC, Ferraz Filho AN, Silva EOR, Lima VLC. Prevalência, indices de infecção e hábitos alimentares de triatomineos capturados em uma área de vigilancia epidemiológica. Revista da Sociedade Brasileira de Medicina Tropical 25:183-190, jul-set, 1992.

12. Rocha e Silva EO, Andrade JCR, Lima AR. Importância dos animais sinantrópicos no controle de endemia chagásica. Revista de Saúde Pública, São Paulo 9:371-381, 1975.

13. Rocha e Silva EO, Andrade JCR, Rodrigues VLCC. Investigação de foco, uma das atividades das campanhas de controle dos transmissores da trypanossomíase americana. Revista de Saúde Pública, São Paulo 12:425-431, 1978.

14. Sherlock IA. Vetores. In: Brener Z, Andrade ZA (ed) Trypanosoma cruzi e doença de Chagas. Editora
Guanabara Koogan, Rio de Janeiro p.42-88, 1979.

15. Silveira AC, Diotaiuti L, Neiva E, Matos CAS, Elias M. Domiciliação do Rhodnius neglectus Lent, 1954 no Estado de Goiás, Brasil. In: Resumos da X Reunião Anual de Pesquisa Básica em doença de Chagas, Caxambu, VE-58, 1983.

16. Siqueira AF. Estudos sobre a reação de precipitina aplicada na identificação do sangue ingerido pelos triatomíneos. Revista do Instituto de Medicina Tropical São Paulo 2:41-53, 1960. 